CORRECTION

https://doi.org/10.1038/s41586-018-0228-6

\title{
Author Correction: Comprehensive molecular profiling of lung adenocarcinoma
}

The Cancer Genome Atlas Research Network

Correction to: Nature https://doi.org/10.1038/nature13385, published online 9 July 2014; corrected online 8 October 2014.

In this Article, the Supplementary Table 7 iCLUSTER output column included incorrect cluster labels for the integrated subtypes presented in Fig. 5c. These changes affect only the iCLUSTER output column and do not affect the analysis or the conclusions of the work. The authors apologise for the error. Supplementary Table 7 has been corrected online, and the original incorrect table is provided as Supplementary Information to this Amendment for transparency.

Supplementary Information is available in the online version of this Amendment. 